\title{
Internet Industry Data Openness and Personal Information Protection Based on Privacy Laws
}

\author{
Yurong Gao ( $\nabla$ simon20130705@163.com ) \\ Huazhong University of Science and Technology \\ Yiping Guo \\ Zhongnan University of Economics and Law \\ Awais Khan Jumani \\ Ilma University \\ Achyut Shankar \\ Amity University
}

\section{Research Article}

Keywords: Industry, Data Privacy, Protection, Law, Internet

Posted Date: September 28th, 2021

DOl: https://doi.org/10.21203/rs.3.rs-924655/v1

License: (c) (1) This work is licensed under a Creative Commons Attribution 4.0 International License. Read Full License 


\section{Abstract}

Data security needs a comprehensive system design approach that combines legal, administrative, and technical protection. These laws generally contain complete rules and principles relevant to the collecting, storing, and using personal information in line with international standards on privacy and data protection. Personal data should be legally collected for a specified reason and not be used without authorization for unlawful monitoring or profiling by governments or third parties. In advocacy and open data activity, increasing attention has been placed on privacy problems. To secure the protection of this data, the Privacy Law (PL) and the Regulations typically put forth industrial and technical standards on IT systems that hold and handle personal data. Concerns about information privacy are genuine, valid, and exacerbated on the Internet of Things (IoT) and Cyber-Physical Systems (CPS). This article suggests that compliance with loT and CPS Data Privacy (DP) at technical and non-technical levels should be dealt with. The proposed architecture is then coupled with a reference framework for the business architecture to offer a DP-loT model focused on the industry and technology and positioned to comply with the Personal Information Protection Act (POPI). Therefore, methods are necessary to protect data privacy based on both system and organizational reference designs. In the end, users should have specific rights to information about them, including the capacity and method to seek recourse to protect such rights, to acquire and amend incorrect details. The DP-IoT model shows a privacy ratio of $92.6 \%$, scalability ratio of 91.5 , data management ratio of $94.3 \%$, data protection ratio of $96.7 \%$, customer satisfaction rate of 92.2 $\%$, attack prevention ratio of $95.5 \%$ and energy consumption ratio of $25.5 \%$ compared to the existing methods.

\section{Overview Of Industry Data Openness And Information Protection Based On Privacy Laws}

An interconnected smart world has become a reality due to the internet's involvement in major industries, allowing for the development of the Internet of Things and Industries, capable of integrating information [1]. The industry is a term used to describe this technological evolution from a manufacturing perspective. To provide a complete solution for integration and integrated industries, it integrates data systems at different stages of industrial processes, involving data exchange both within and across industries and converging information technology systems at different levels of an organization [2]. Through the development of intelligent goods and processes that can enhance traditional business models, cyber-physical systems, these features promote the smart industrial approach [3]. This can influence industrial processes through Information Technology (IT) convergence to promote connected factories by integrating the Internet of Things and Industries with CPS [4]. As a result, a standard design framework is adopted to offer technology solutions and laws for integrating and managing effective technologies [5]. It has been widely discussed how CPS and other technology developments can be implemented in the fourth industrial environment to integrate and enable interoperability between different industry sectors [6]. 
Industry can establish a significant advantage through smart manufacturing, improved decision-making and data analytics via implementing loT technology in the industry [7]. When it comes to important facilities, the industrial loT is mostly used for monitoring and controlling them. Intelligent transport, smart cities and device-to-device connectivity are instances of industrial loT applications [8]. Security and privacy mechanisms must be in place to ensure safe and efficient functioning. The loT industry is defined by time constraints and major safety and economic losses [9]. While industrial loT can be targeted with the same kind of privacy attacks as consumer loT, there is a difference in the impact of such attacks when they are effective [10]. An unauthorized device connecting to a consumer loT network, a loss of data privacy and data theft, and a similar attack on a typical loT industry network can result in a significant massive failure, such as the network being interrupted and being made to participate in a dangerous activity [11]. A higher level of privacy is required for industrial loT, considering timeline demands, types of devices in the network, recovery in case of attack, and similar factors. That's not common for third-party cloud services for data storage and analysis in industrial loT applications [12]. As a process, a new system vulnerability is created. As a result of the loT being integrated into industrial processes with higher privacy laws, various industry standards, device production frameworks, communication protocols, privacy services, and technologies have been developed and offered to industry [13]. This article evaluates privacy protection in industrial loT communications designs related to industry framework needs and present connection technologies. From a business perspective, the study analyzes the current state of industrial privacy nowadays [14]. It is proposed to evaluate this industry's research and includes possible industrial data privacy [15]. The Internet Industrial development approach gives detailed information on the functions that cyber-physical systems perform at various levels of loT [16].

A technology distributed at the edge devices is classified under the functional viewpoint, and technologies that bridge connection are categorized under the implementation and information perspectives [17]. This describes the loT industry design styles that describe privacy properties and how information is connected from a consumer perspective. Each component of the system from the edge to the industry needs privacy capabilities [18]. As a result, data passing across the various levels can be continuously protected against unwanted attacks and eavesdropping to remain private [19]. According to the internet industry trends, an loT network can implement authentication protocols that provide a balanced approach at the endpoint level. Enable edge-to-cloud connection that is cryptographically protected [20]. Quantum-resistant encryption suites can be used to improve the privacy of data transmission. Effectively protect data using hardware privacy modules Provide interoperability across systems from different industries [21]. In evaluating the privacy risk of data loss centers, physical security of connectivity, network protection, and data flows can be addressed [22]. Protecting data exchange and connectivity in loT industry systems requires information protection, monitoring, analyzing data transmission, establishing security mechanisms and developing privacy laws for data communication and connectivity. Authentication, access control, capacity management, load balancing, and storage are several methods that can be used to provide privacy at various levels $[23,24]$.

\section{The main contribution of this article,}


A complete analysis of industrial loT networks is provided in this article. Thus it provides recommendations for data protection and assesses relevant data privacy at various layers in connection to the established requirements. This study explores how secure connectivity can be maintained in the loT industry using a detailed view of the industry and an analysis of how network protocols interact. Identify potential privacy vulnerabilities that can occur at various stages and address what has to be done. Using data privacy laws systems, this article identifies the challenges of personal information protection in the loT. Given information protection and examines current advances in the loT.

The remaining structure is organized as follows: Section 2 describes the literature work and its corresponding discussion. In section 3 , the proposed method DP-loT is discussed. Section 4 summarizes the numerical results compared with an existing method. Section 5 concludes based on the previous section's analysis and discussion.

\section{Literature Study}

Fataliyev T. et al.[25] described the Internet of Things, cyber-physical systems, artificial intelligence, and other advanced technologies closely related to Industry 4.0. It was accompanied by an increase in data volumes in Industry 4, which brought important data protection difficulties to the fore. Using personal data in unauthorized ways to acquire additional preferences has become a goal for certain organizations. The privacy of private information processed in surveillance systems was given greater attention since video surveillance data are essential to personal data. Information communication networks for transmitting data were included in the monitoring system, processing equipment, analytic software, and personal data storage devices.

Fernández-Caramés, T. [26] evaluated the models driving the modern industrial revolution by connecting industrial devices and management tools to the internet to control and gather data about them. A large number of loT industry devices were related to the internet without considering suitable security measures, resulting in a large number of devices that were misconfigured and had insufficient protections in place. It was explained in this article how Industry and IloTcybersecurity could be introduced through practical examples, using an auditing method that allows students with no existing expertise in loT and industrial cybersecurity to be evaluated. As a result, this article provides valuable suggestions for addressing industrial cyber security in the next generation of security research communities.

Patel T. et al. [27] introduced building Information Modeling (BIM) as a digital technology that replaces paper-based data interchange in the construction industry. In the Construction industry, an increasing amount of personal and sensitive data was being digitally collected and stored in the cloud, making it vulnerable to cyberattacks. Secondly, it's important to develop a framework and strategy for protecting construction data privacy. The law should protect the addresses of individuals and any other additional information. In this method, they use a randomization technique to modify the original data and then use privacy protection to the changed data to improve accuracy with minimal information loss. Data must be 
kept anonymous, which is achieved by the privacy-preserving data mining approach (PPDMA). By implementing this approach, private data would be protected from cyber-attack by mining techniques.

Sharma S. et al. [28] initialized that more duplicate data was sent over the internet due to loT devices' growth. There were layers of loT devices used to detect data to prevent security threats in this study and activity allocation and secure duplication were performed. An elliptic-curve cryptography-based hybrid multiplier (ECC-HM) was used to connect the devices in this layer with the cloud server. Service-layer search results for loT users would be available. Users' satisfaction, network durability, and security had improved due to the research findings.

Kounoudes, A. et al. [29] suggested increasing issues about privacy protection in the Internet of Things due to the large quantity of personal data gathered and exchanged by the loT (Internet of Things). As a result of the new general data protection regulation (GDPR), customers' privacy rights were increased and new standards for data handling were established. Influencing this study was the results of existing research in this field, which revealed that GDPR requirements in loT were challenging to implement because of technology constraints. Using the research as a guideline, this finding examines the state-ofthe-art how these characteristics are addressed. Lastly, they analyze their findings and identify open challenges in privacy protection that require future investigation by the research community to be handled.

Latif, S. et al. [30] demonstrated a major role in the industry was performed by the loT technology, a collection of secure, accessible, and widely recognized technologies being implemented for the smart industry. A single point of failure and many cyber-attacks were common in traditional loT architectures, making them vulnerable to loT design flaws. This ideology proposes a blockchain-based architecture (BC$\mathrm{BA}$ ) for industrial processes that was secure and dependable. To manage access to important sensors and control data, a private and compact blockchain-based architecture was suggested. The suggested framework for smart industrial settings was demonstrated success through extensive experiments and research. They converted a traditional product processing facility into a secure and smart industrial platform by applying the suggested design.

Based on the survey, some existing PPDMA, ECC-HM, GDPR and BC-BA methods demand improved privacy, scalability, data management, data protection, customer satisfaction, attack prevention, and energy consumption ratios. Therefore, this study presents the DP-IoT model that can support the personal information protection act (POPI).in the industry as detailed below.

\section{Dp-iot For Industry And Positioned To Comply With The Personal Information Protection}

The Information protection act ensures security and data privacy for industries. This involves implementing industry standards in data privacy and security, collecting customer information with the implied contract of customers, and providing access to their data once their authorization has been 
acquired. Industries can effectively protect, manage and improve loT implementations with internet mobility platforms. For this purpose, this article has established skills and abilities to reduce risks by developing loT applications safely. In addition, it is focused on collecting personal information to respect the privacy and security of its customers' personal information. Industry security is responsible for loT devices and data processing activities connected with loT management, as industries have to process personal information. Security and privacy are integrated into the design of industrial products, as is the possibility to include data to these rights into business development. Integrating data privacy measures into loT systems and data processing is one approach to achieve this. The high Internet adoption and data location make it a perfect target for additional privacy law considerations. Authorities in Asia discuss improving their privacy laws, such as the Information Protection Act and data privacy. This article has to update the information protection standards to take into account loT's complexity and implications. The loT model is challenged by limitations on data collection and free usage, such as storing data in the industry. Although loT technologies such as sensing devices are integrated into the network and often placed discreetly, it can be difficult to acquire individual customers' authorization. After all, various industries, such as cloud services, are involved in the collecting and transmitting data, and third parties usually extend their rights to privacy and information protection.

The large majority of connected devices do not sufficiently disclose how their customers use their personal information. As loT devices continue to be implemented, a lack of awareness about their privacy and security impacts and use. Given the extent to which Internet of Things (IoT) industries include collecting, processing, and storing personal information such as credit card details and bank account data, such failures cannot result in a huge revelation. Compatibility with the DP is heavily difficult in the Internet of Things as it can be challenging to get the essential authorization to process personal information on an loT network. Establishing the data controller and processor for an loT-related processing activity can be a major challenge. IoT devices are rarely aware of this supporters are working to change this under the general data privacy law. Customer satisfaction can be a distinguishing characteristic of IoT industrial companies that are particularly devoted to data protection. According to the loT, huge amounts of data are created that can be processed and analyzed in various ways, including in real-time and without the need to get authorization. Due to this inflow of information is useful to assess data privacy that resulted in the creation of the information protection act. The data privacy law is essential to protect personal information by setting a standard for other countries and regions.

Figure 1 shows the data security attacks in the loT industry. Several users have mentioned the assault of Internet-connected devices predicted to be a reality for several years. This interconnectivity can lead to incredible productivity, effective industry systems and efficiencies that are unachievable. It can provide tempting possibilities for a digital world of hackers and other fraudulent activity aiming to get to digital security systems that did not exist before the palace's area has become low. Therefore, it is simple to understand why the interconnected devices of the future have to be developed with privacy from the initial stage. More than $25 \%$ of cyberattacks can include loT devices, giving vulnerabilities a critical problem. Having laws can protect consumers and enabling this change to occur. These systems are on their approach to becoming essential parts of today's consumer existence. Therefore data can be 
protected. Minimal, purpose-specific loT devices are the objective of loT devices. Customers are using them to collect data, depend on it and use connectivity as designed. As a result, an extra privacy device of that capacity is still in its development. If only one loT device in the network is found to have a vulnerability, it can have a domino impact on the entire network of loT devices. loT is the distribution of connectivity to a physical object. As it develops, this digital existence can initiate interaction and transfer context data, and the devices can make decisions based on this interactional device. The loT is the allocation of connectivity to a physical object. In the end, this can create physical threats to network security, personal possessions (such as automobiles and homes), and the environment such as power, water and food. In addition to power and energy, exploration and production, industrial and chemical plants, healthcare and aviation are covered by the loT technology. Since loT technology has been widely established worldwide, hackers have a better time accessing these devices. There's no solution to let the Internet of Things turn into the Internet of Threats. Security and data privacy are the main categories under which loT threats can be classified.

IoT devices can be protected from attacks that could affect the efficiency of devices. Since devices can transmit significant amounts of private data over the internet, and end-users can effectively support a device, data protection can be integrated into each systems level of the network. When it comes to operating loT devices in a secure environment, its complexities have increased. This increasing complexity creates security problems beyond the challenges consumers encounter when protecting a single device according to their own. It is suggested that difficulties related to information protection, privacy and physical security are connected and that solutions that will solve four aspects simultaneously are presented. To create data protection requirements for personal devices, technology is used.

Further implementations of such systems can be influenced by analysis that establishes the optimum approach for customers to handle collections of devices securely. Integrated device industries can be used to protect customer data. Since an attack can occur, each subsequent network within the interconnected fortress must be protected against it. loT systems can be improved highly secure using data encryption such as data protection, effective user authentication, adaptable development, established and verified protocols, although attacks can constantly occur. For loT systems to be secure, authentication, data evaluation, and data security encryption are required. With better code development standards, threat analysis, and validation, software development industries need to improve their capacity to produce secure, durable, and trustworthy technology at the application level. As a result, the implementation of secure and effective integration standards is important.

In an environment when more and more smart devices collect data, transmit it, and profit from it, privacy is challenging to identify and maintain. The significantly different data privacy laws constrain the loT's success. The data collected by loT devices encourages loT customers to spend additional attention on what happens to the data created by loT devices. These devices are placed on the internet for efficiency, causing great public discomfort at the minimum and significant invasion of privacy in some situations. In the maximum, devices conduct large-scale Internet attacks to connect to personal information such as 
location and activities. As a result, there are challenges about how to protect the privacy of personal information. The idea of the authorized startup is one of the main aspects given out by the cloud security interaction and most others in the security industry. A device's core is the technology that it processes therefore, this application can be tested to ensure it is operating correctly before it can be used. It's possible that the device's code can first measure itself and then other code modules while transferring control to other code modules. The industry's solution for data privacy is to evaluate code before processing to address an initial comment. Using security and privacy, this study explored some of the consequences of these new developments. Consider that changes occur rapidly and the level of uncertainty is such that focusing investments in technology to reduce possible concerns is the highest priority for industries and research equally. Considering the potential benefits to human existence, public security, and the economy, major research efforts are required to increase the technology's usefulness. Authorized data industries, in specific information traders, do not have regulatory norms. There is a lack of openness regarding who gets access to data, how it is used to produce goods and services, and how it is transmitted to customers or other third parties. Data storage, usage, and privacy, including data, can be controlled by clear laws.

Figure 2 illustrates the components of Industry 4.0. As part of a private Industries 4.0 initiative, new technologies can be developed in the industrial environment by integrating physical, digital, human, and biological worlds. These foundations include the Internet of Things (IoT), Industrial Internet (II), and the integration of these technologies in the production process. loT concepts and technologies and an industrial network of sensors and complicated industrial devices and controllers can be created, connected to information techniques that enable the systems to monitor, analyze, distribute, collect, and change data efficiently in real-time. In this way, the fourth industrial can achieve its main objective: open innovation through value networks to support its business objectives. Integrating digital, physical worlds across a production process and distribution channels by connecting industrial systems is the objective of end-to-end innovation. It suggests that the integration of information and operational technologies loT can produce many benefits for industrial environments. Data storage, processing, and transmission are being integrated into production control systems in the information technology industry. Hardware and software systems are part of information technology, typically integrated into a more extensive digital system for industrial processes. According to the Information and operational technologies integration, industrial machines now can communicate directly with other devices and centralized databases, exchanging data over the network and reducing the number of required activities, improved performance, higher asset usage, resource cycle cost minimization, and better decision making, purchase and sales. These products as activities improve industry opportunities and enable the development of new industrial business models. Thus it is important to understand the demands for industries that can create networks of assets, storage systems and production processes in Cyber-Physical Systems (CPS). The organization proposes CPS as one of the key technologies of industry. CPS contains a lot of challenges that are included in loT.

An interactive environment in the industry can be created by integrating network technology, computing, and storage. Smart devices having private keys that can be located in real-time have become standard in 
this approach. This distributed system integrates physical reality, communication networks, and technological resources into a single, automated system. Although conventional embedded systems focus on a single device, industrial embedded systems network a wide variety of devices. Given that devices and sensors interact with the physical surroundings, the system includes a management system capable of handling interactions. The CPS can be described as a collection of systems capable of sending and receiving data from devices through the internet and a network. Component of a centralized production system, industrial devices are connected to the internet in real-time, independent of location. With real-time connectivity, it's essential to show stability, efficiency and effectiveness. To protect private information and ensure data privacy, the industry aims to provide security support at each level of the CPS design. CPSs are used in the fourth industrial environment to ensure a proper interface across automated processing areas. Auto monitoring, production control and data exchange in real-time are used in industry. Several new systems have been established due to the fourth industrial is increase data and industrial devices. These include proposals for CPSs, fourth industrial production for device virtualization in the production process, loT integration and interaction.

Figure 3 shows implications for the Internet of Things. The Internet of Things can significantly influence various aspects of a customer's daily interactions and the local environment. According to Figure 3 , life quality can increase in multiple areas such as energy, security in the workplace, the environment and healthcare. Although loT devices are resource-constrained in processing, storage capacity, connection, and network protocols, this is not a disadvantage. Limitations such as these have a direct impact on the type of technology that is used to protect data privacy. Promoting loT adoption between customers and reducing the risk of data theft and privacy. loT devices collect personal information, such as the customer's name and location, energy consumption, and telephone numbers, regarded as a data protection threat and a significant privacy problem. Data on customer's everyday activities, such as using home appliances, watching TV, leaving and coming to their homes, can be collected from this data. The fundamental problem is that these devices can collect private information from customers and control their environments. This poses a serious threat to security. A lack of trust in public and private databases makes users uncomfortable with exposing their personal information.

Consequently, the lack of loT privacy techniques could prevent customers from implementing any loT technology. Customer privacy loss and associated threats are illustrated in the following points. Lots of studies have been focused on loT privacy and security concerns, difficulties and solutions. Classifying privacy threats and identifying problems in loT scenarios are its primary goals. This study introduces industrial loT privacy challenges. A complete and secure environment for industrial loT is discussed and possible solutions to these complications.

This research focused on security issues and current solutions. For example, it categorizes the challenges: authentication, access control, data encryption, privacy and trust. There are several physical resources in the loT device interface. These are highly variable and limited in terms of the resources available. Such constraints complicate the application of privacy-preserving methods. As a result, loT devices are vulnerable to many attacks, including network captures, false networks, secret 
communications, timing attacks, routing threats, replay attacks, side-channel attacks, and large node authentication difficulties, are covered. Authentication and access control measures to protect customer privacy from unauthorized access. Secure data transmission and delivery are maintained by data encryption. A nonlinear key algorithm based on displacement computation is presented to offer data encryption. As a result of its low computing requirements, this essential process provides excellent privacy and high data transmission rates. Privacy protection, security, authenticity, and data management are some of the benefits of encryption technology. The objective is to be aware of private information gathering, threats, and how to use loT services in a secure environment. loT privacy is discussed, including encryption techniques, communication security, sensor data protection, and encryption algorithm to ensure privacy in loT. Given the fact that security and privacy are closely connected, the concept mainly focuses on privacy issues. A wide range of privacy-related aspects in open loT settings is addressed, allowing for greater insight into design principles and the development of private information IoT settings. As a result, privacy preservation is a shared responsibility that requires industry leaders to participate actively and collaborate to ensure secure loT environments while appreciating what loT has to provide. Manufacturers of IoT devices have to integrate privacy and security protections into their designs to ensure industry development. loT-oriented procedures can be included in facilities to avoid data losses and handle security threats from the bottom up. Customers of loT applications can be made aware of what data is being gathered and why. Those who use the Internet of Things (IoT) can exercise additional awareness when accessing their private information and better understand the potential implications if that data is mishandled either way.

Fig. 4 shows the design of the industrial Internet of Things loT. A basic loT architecture is composed of devices, networks, clouds, and applications. As a closed environment, it creates specialized and customized products to meet the demands of the end-customer. Observation, network, process and application layers are the four levels of the systems. Various sensors, security devices, and GPS units contribute to the observation layer. These devices can be supported by technology such as transportation systems and industrial automation in an industrial environment. These devices are responsible for collecting sensory data, monitoring environmental conditions, and distributing goods. WiFi, Bluetooth, and $3 \mathrm{G}$ are network layer elements that use IPv4 and IPv6 to transmit data to the processing layer system. Several functions can be accomplished by the Data layer, including decision making, performing optimization methods, and collecting large amounts of data. Application layers are responsible for providing industry customers access to application-specific capabilities. Smart home, smart city, EHealthcare, Vehicular Ad-Hoc Network VANET are important loT-based applications.

In contrast, smart grid, fourth industry, smart devices, production lines, transforming warehousing are considered loT applications. loT network architecture with centralized control has the following disadvantages, to summarise. As a result, the entire network can become paralyzed. For this, the data stored on centralized databases can be mishandled by customers who have no access to their data. Centralized data can be changed and deleted. 
There is no security of accountability and traceability of the data stored on the databases. A centralized customer's inefficient handling of large amounts of end-to-end interactions can significantly constrain loT development. Due to the general explosive growth of the loT (IoT), effective security and privacy laws can be implemented to prevent system vulnerabilities and attacks. The loT has various other important considerations, such as dependability, scalability, and energy consumption. The standard security products are not necessarily appropriate in this environment. Due to these considerations, the study's main aim is to evaluate the different security threats classified by loT-related objectives. People can benefit from this object-based classification system to establish which attacks are important to their specific application, research, users, and industry. In addition, customers can analyze solutions and develop new systems based on the applications they are focusing on. The implications of specific loTrelated attacks can be significantly serious in an loT industry environment. A repeat attack on industrial loT device transferring materials from the warehouse to the production floor, for example, can result in a stoppage of the production floor production line. In this way, the researcher summarizes how traditional privacy issues and the most critical threats are addressed in the Industrial Internet of Things domain. Generalized industrial loT project requires third-party industry to provide security and privacy advantages, and customers exchange personal information with these entities. Due to its private and distributed design, digitalization presents a potential solution to the above challenges. As a distributed ledger, a network consists of timestamp blocks connected by private keys. The integration of digitalization has provided answers to many of the significant issues faced by generalized loT designs addressed in this survey.

\section{Algorithm1. Data access control}

Input: Question set $Q_{s}\left(Q_{s} 1, Q_{s} 2 \ldots Q_{s j}\right)$ Where $i$ is the total number of questions.

Output: The result of something (number of rows).

Start

// User names and passwords should be provided for this search to function.

Then, if (Result $==1)$ is true, Limit the outcome

\{

Else if (Result $>=0$ ) then \{

// shows the number of rows in a query result.

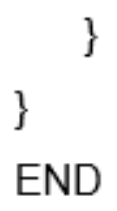

Figure 5 shows the flow chart for the data access control algorithm. Simulated to manage data access and sharing, this algorithm controls data access and sharing. The internet connects every system. Data analysis can be a registered and unauthorized user. Laws have been established for request answers in this algorithm. A unique username and password can be assigned to each database platform by the 
system. As a result, Algorithm 1 can be used to regulate data access. When an unauthorized individual attempts to retrieve the data, it is controlled by the access control algorithm used.

\section{Result And Discussion}

Essential aspects of the Internet of Things include developing security data analytics techniques to deal with failures and effective attacks. Due to the massive loT's ability to support industrial systems based on time-sensitive information, real-time data transmission and management, any privacy law can offer protection services and preserve the timeliness of data transmission. loT systems in the industry require data. Therefore it stands to reason to store it in a third-party database management system. This involves serious issues concerning the integrity of the information under the control of a third party, and there is a need to maintain privacy to protect valuable industrial assets from outsiders. Further research is needed on scalability, quick and secure data transmission from the internet in time-critical applications, and effective data recovery when third-party storage systems have been hacked. In many industry loT applications, the hardware and software are mixed, providing additional research challenges. As data flows at several levels and an interacting collection of hardware and software, it's challenging to provide the privacy protections required. Significant demand in end-to-end encryption is essential as data flows across numerous hardware platforms from different industries and software and privacy protocols from different vendors. In loT implementations where an attacker can actively influence a device, development into creating encryption devices and maintaining security in the presence of such physical attacks can be addressed. Identifying and recovering from device-capture attacks is a significant challenge in the loT since an attack on one device can damage a whole system. Appropriate data privacy laws can be sufficient in protection applications. IoT connectivity privacy is a difficult challenge to solve. For a complex loT system to have a set of unique security requirements, extra research is required. - When it comes to privacy, data collection can be done to protect customer's personal information. Data from source to destination gathered data is one technique to accomplish this. A device's identity can need to be verified by requesting it to prove itself without disclosing information. As a result, techniques for privacy protection and the lowest verification can be studied and developed for the loT in the industry.

Table1. DP-loT outcomes 


\begin{tabular}{|llllll|}
\hline Number of Customers & PPDMA & ECC-HM & GDPR & BC-BA & DP-loT \\
\hline Privacy Ratio (\%) & 48.3 & 53.4 & 76.1 & 82.6 & 92.6 \\
\hline Scalability Ratio (\%) & 55.1 & 65.5 & 71.2 & 83.7 & 91.5 \\
\hline Data Management Ratio (\%) & 54.2 & 68.3 & 75.4 & 86.5 & 94.3 \\
\hline Data Protection Ratio (\%) & 55.9 & 64.7 & 75.5 & 85.7 & 96.7 \\
\hline Customer Satisfication Rate (\%) & 58.4 & 66.5 & 77.4 & 87.9 & 92.2 \\
\hline Attack Prevention Ratio (\%) & 63.0 & 73.6 & 81.9 & 89.5 & 95.5 \\
\hline Energy Consumption Ratio (\%) & 83.4 & 66.5 & 53.4 & 37.9 & 25.5 \\
\hline
\end{tabular}

Table 1 shows the DP-loT outcomes. loT technology is increasingly becoming an essential part of many industrial processes in industries and protection control applications. As a result of its various hardware and software, complicated subsystem interactions, and high privacy requirements, the loT is essentially difficult to understand and use. Given the complexity of the systems, and since it is difficult to explicitly describe privacy needs and implement them effectively in a way that can be proved, maintaining loT security and privacy is challenging in industries. Using suggestions from well-known standards groups, the study aimed to give a comprehensive perspective of data privacy in the loT such this search can identify privacy protocols. Different privacy laws and solutions have been reviewed, focusing on identifying information protection flaws and vulnerabilities. loT privacy issues are discussed and proposed approaches for research to overcome issues.

\section{i) Privacy Ratio (\%)}

As devices analyze and transmit huge volumes of data, loT applications can compromise the customer privacy required to maintain competitive advantage if data are not collected, processed and exchanged securely. Maintaining privacy is a complicated challenge that involves both legal and technical challenges. Industrial operations, such as factories and protection control applications, are increasingly adopting the loT. Assuring privacy in Industrial loT systems is difficult due to the complexity of the systems and the challenge of describing secure requirements and implementing these in a method that can be proved.

The privacy ratio (\%) is shown in figure 6. A privacy-preserving technique of data collection and processing can be used to improve privacy. As a last effort, devices can be requested to verify their identity without sharing data. There has been a detailed analysis of various privacy processes and solutions, focusing on identifying privacy vulnerabilities and threats. The study suggests alternative paths for additional research to address some of the privacy issues in industry loT.

ii) Scalability Ratio (\%) and Data Management Ratio (\%) 
It is essential to integrate appropriate privacy laws to prevent any vulnerabilities in systems from developing. Another important problem with loT technology is reliability, scalability and energy consumption. Internet capabilities allow industry loT to introduce additional facilities at any time. As the number of loT devices increases, the amount of data is collected, requiring higher storage capacity to keep the database updated. In addition, this can result in high processing and storage costs. IoT scalability improves as the number of customers and transactions improves. When a particular transaction size is achieved, latency between placing a transaction and authenticating it starts decreasing. It enables more automation, scalability, and lower transfers (there is no need for a third party to monitor transactions), which can help avoid data exploitation by people. To increase scalability, this article proposes maintaining a separate database depending on geographical location.

Figure 7 (a) shows the scalability ratio (\%) and data management ratio (\%) are shown in figure 7 (b). As data became easier to acquire, various risks and crises involving data security developed. This article can effectively plan for data security measures to ensure data security and utilize technology's efficiency and benefits. Three requirements for data security management include protecting private information connected to telecommunications industries from unauthorized access. The use of communications infrastructure can be highly regulated to ensure authenticity. To provide management support and guidance for data security by the industry's requirements and relevant laws, a data management document can be approved by management communicated to the employees and the entire industry. For data security events to be quickly resolved effectively, management responsibilities and methods can be defined. Any observed and suspected theft, leakage and modification can be recorded through the proper management channels, resolved and prevented from further industry damage. Customers' trust in the industries can be evaluated by a robust control system and active management.

\section{iii) Data Protection Ratio (\%) and Customer Satisfaction Rate (\%)}

Products and services from the Internet of Things are eventually shifting their focus into people's daily lives. As a result, personal data protection becomes a highly important aspect. Devices and industries can be reused in a wide range of environments. Identifying the appropriate solutions for personal data protection can be significant. Data protected by privacy law are essential as if data are exposed, it can provide a key for malicious persons to access the personal data of consumers and industries. To find the optimal solution for data protection, the characteristics of the industry to be offered can be considered. Having a system in place to protect personal data could significantly influence the customer and the industry. The suggested system aims to find solutions for protecting personal data acceptable for a given loT service. Various industry variables can influence the effectiveness of the solution in some situations. Finally, the industry can receive a legal authority given to the industries and its customers. There are legal industries that can assess how personal data protection laws can change current industries.

Figure 8 (a) data protection ratio (\%) and customer satisfaction rate (\%) are shown in figure 8 (b).Customers have been provided with the best quality of service data network, suggesting that the system has improved its performance. As a result, user customers satisfaction is determined by 
considering each quality of service factor. As shown in Fig. 7, a variety of loT devices have high customer satisfaction. In industrial applications, this measure influences the quality of service parameter's efficiency as customer satisfaction is important, and the design provides secure storage and task allocation for industrial applications. Customers are satisfied with the proposed approach as it reduces processing times for activities while maintaining the highest level of customer satisfaction achievable. The customer satisfaction rating is lower as cloud servers are poorly managed. Data storage is essential to manage the services that loT customers can access and maintain data in a proper order that can be handled. For this, it takes a long time and in some circumstances, data can be accessed incorrectly by customers. As a result, customer data is transferred to a data center, which reduces delay and increases service quality and the proposed system has improved customer satisfaction.

\section{iv) Attack Prevention Ratio (\%)}

The proposed authentication technique is difficult to repeat attacks since the identity of a device (used in authentication) is generated based on a copy of a random quantity unidentified to an attacker. Similarly, the authentication protocol requires three random integers for the customer, node, and gateway around every interaction. This prevents various attacks by ensuring that communication messages have been updated. Since this private key is stored in the data sets, an attacker cannot calculate the data packet even if they can get the device's identification.

Table2. Attack Prevention Ratio (\%)

\begin{tabular}{|c|c|c|c|c|c|}
\hline $\begin{array}{c}\text { Number of } \\
\text { Customers }\end{array}$ & PPDMA & ECC-HM & GDPR & BC-BA & DP-IoT \\
\hline 10 & 53.7 & 63.7 & 74.7 & 82.4 & 89.7 \\
\hline 20 & 54.8 & 65.8 & 75.8 & 82.5 & 90.8 \\
\hline 30 & 55.7 & 66.7 & 76.7 & 83.7 & 90.7 \\
\hline 40 & 56.7 & 67.7 & 77.7 & 84.9 & 91.7 \\
\hline 50 & 57.9 & 68.9 & 78.9 & 85.3 & 92.9 \\
\hline 60 & 58.8 & 69.8 & 78.8 & 86.1 & 93.8 \\
\hline 70 & 60.6 & 70.6 & 79.6 & 86.3 & 93.6 \\
\hline 80 & 61.8 & 71.8 & 78.8 & 87.9 & 94.8 \\
\hline 90 & 62.5 & 72.0 & 80.9 & 88.5 & 94.1 \\
\hline 100 & 63.0 & 73.6 & 81.9 & 89.5 & 95.5 \\
\hline
\end{tabular}

Table 2 shows the attack prevention ratio (\%). Furthermore, the attacker cannot appear as a trustworthy network since the pre-shared key isn't available to the attacker. Customers can be protected from Man-in- 
the-Middle attacks using the user authentication technique mentioned. An attacker needs several secret parameters to transmit, receive, and alter the authentication process, such as the customer's and sensing device's secret Identities. In such an environment, protection methods are important, and data storage can help by providing suitable authentication mechanisms, hardware and software updating processes that can help improve security. As a result, authorized customers can have access to the network, while nonauthorized customers cannot. Without authorization, malicious users can gain control of personal data and access it.

\section{v) Energy Consumption Ratio (\%)}

In loT devices, energy consumption is a major challenge. However, industry loT sensors and devices are energy-constrained, whereas some processes demand a huge amount of energy to operate at maximum capacity. As a solution to this issue, efficient energy activities such as clustering can be proposed. As a result of clustering, $\mathrm{CH}$ transmits data to neighboring cloud servers, reducing the need for computation and enabling loT devices to reduce energy consumption.

\section{Table3. Energy Consumption Ratio (\%)}

\begin{tabular}{|llllll|}
\hline Number of Customers & PPDMA & ECC-HM & GDPR & BC-BA & DP-loT \\
\hline 10 & 92.6 & 75.9 & 65.2 & 50.2 & 36.8 \\
\hline 20 & 91.6 & 74.7 & 64.7 & 48.3 & 35.7 \\
\hline 30 & 90.3 & 73.6 & 63.4 & 46.6 & 34.4 \\
\hline 40 & 89.1 & 72.9 & 62.1 & 45.9 & 33.5 \\
\hline $\mathbf{5 0}$ & 88.7 & 71.3 & 61.7 & 44.5 & 32.7 \\
\hline $\mathbf{7 0}$ & 87.6 & 70.2 & 60.4 & 43.4 & 30.2 \\
\hline $\mathbf{8 0}$ & 86.7 & 69.2 & 59.5 & 40.6 & 29.3 \\
\hline $\mathbf{1 0 0}$ & 85.9 & 68.8 & 55.5 & 39.2 & 28.6 \\
\hline
\end{tabular}

Table 3 shows the energy consumption ratio (\%). According to the graph, the proposed method has resulted in a reduction in energy consumption. Data centers verify data deduplication at random, using cloud networking. Significant computations are required to transmit data requests from devices to edge devices, resulting in increased energy consumption at loT devices. These studies offer work distribution and secure processing using the internet's highest identification and convolution layer to reduce energy consumption in industry loT. 
The proposed method shows the privacy ratio, scalability ratio, data management ratio, data protection ratio, customer satisfaction rate, attack prevention ratio and energy consumption ratio when compared to privacy-preserving data mining approach (PPDMA), An elliptic-curve cryptography-based hybrid multiplier (ECC-HM), general data protection regulation (GDPR) and blockchain-based architecture (BC-BA).

\section{Conclusion}

In addition, privacy law can be created the connection between personal information protection and authoritarian management that security forces can implement can be carefully maintained and secured. As a result, this idea is optimistic that local authorities and industry leaders can establish discussions and laws to protect customers' loTby identifying industry trends from enhancing consumer information protection in the loT. During this year, the industry released a series of quality standards and privacy laws. The development and implementation of privacy laws for loT devices that helping to organize.loT, the privacy issues it creates, and the importance of implementing information protection for loT devices and industries in a secure. This study concluded with new research objectives, challenges and potential work on privacy, scalability, data management, data protection, customer satisfaction, attack prevention, energy consumption and real-time search that enable loT to provide information protection with privacy law approaches in the industry.

\section{Declarations}

\section{Ethics Declarations}

Conflict of interest

The authors declare that they have no conflict of interest.

Ethical approval

This article does not contain any studies with human participants or animals performed by any of the authors.

\section{References}

1. Masud M, Alazab M, Choudhary K, Gaba GS (2021) 3P-SAKE: privacy-preserving and physically secured authenticated key establishment protocol for industrial wireless networks. Comput Commun 175:82-90

2. Abdel-Basset M, El-shahat D, Elhoseny M, Song H (2020) Energy-Aware Metaheuristic algorithm for Industrial Internet of Things task scheduling problems in fog computing applications. IEEE Internet of Things Journal

3. Sivarethinamohan R (2021) Behavioral Intentions towards adoption of Information Protection and Cyber security (Email Security and Online Privacy): SEM model. Turkish Journal of Computer 
Mathematics Education (TURCOMAT) 12(6):56-68

4. Shakeel PM, Baskar S, Fouad H, Manogaran G, Saravanan V, Montenegro-Marin CE (2021) Internet of things forensic data analysis using machine learning to identify roots of data scavenging. Future Generation Computer Systems 115:756-768

5. Lv Z, Han Y, Singh AK, Manogaran G, Lv H (2020) Trustworthiness in industrial loT systems based on artificial intelligence. IEEE Trans Industr Inf 17(2):1496-1504

6. Heide M, Villeneuve JP (2020) From secrecy privilege to information management: A comparative analysis of classification reforms. Government Information Quarterly 37(4):101500

7. Sutrala AK, Obaidat MS, Saha S, Das AK, Alazab M, Park Y (2021) Authenticated Key Agreement Scheme With User Anonymity and Untraceability for 5G-Enabled Softwarized Industrial CyberPhysical Systems. IEEE Transactions on Intelligent Transportation Systems

8. Cao Y, Jia F, Manogaran G (2019) Efficient traceability systems of steel products using blockchainbased industrial Internet of Things. IEEE Trans Industr Inf 16(9):6004-6012

9. Jia L, Ruan L (2020) Going global: Comparing Chinese mobile applications' data and user privacy governance at home and abroad. Internet Policy Review 9(3):1-22

10. Seyhan K, Nguyen TN, Akleylek S, Cengiz K, Islam SH (2021) Bi-GISIS KE: Modified key exchange protocol with reusable keys for loT security. Journal of Information Security Applications 58:102788

11. Gao J, Wang H, Shen H (2020, May) Smartly handling renewable energy instability in supporting a cloud datacenter. In 2020 IEEE international parallel and distributed processing symposium (IPDPS) (pp. 769-778). IEEE

12. Mangku DGS, Yuliartini NPR, Suastika IN, Wirawan IGMAS (2021) The Personal Data Protection of Internet Users in Indonesia. Journal of Southwest Jiaotong University, 56(1)

13. Manogaran G, Alazab M, Shakeel PM, Hsu CH (2021) Blockchain assisted secure data sharing model for Internet of Things based smart industries. IEEE Transactions on Reliability

14. Sah DK, Nguyen TN, Cengiz K, Dumba B, Kumar V (2021) Load-balance scheduling for intelligent sensors deployment in industrial internet of things. Cluster Computing, 1-13

15. Chen J, Zheng Y (2020) User information protection of e-commerce platform business based on credit evaluation system. IseB 18(4):945-954

16. Gheisari M, Najafabadi HE, Alzubi JA, Gao J, Wang G, Abbasi AA, Castiglione A (2021) OBPP: An ontology-based framework for privacy-preserving in loT-based smart city. Future Generation Computer Systems 123:1-13

17. Amudha G (2021) Dilated Transaction Access and Retrieval: Improving the Information Retrieval of Blockchain-Assimilated Internet of Things Transactions. Wireless Personal Communications, 1-21

18. Fataliyev T, Mehdiyev S (2020) Industry 4.0: The oil and gas sector security and personal data protection. International Journal of Engineering Manufacturing 10(2):1

19. Manogaran G, Alazab M, Saravanan V, Rawal BS, Shakeel PM, Sundarasekar R, ... Montenegro-Marin CE (2020) Machine learning assisted information management scheme in service concentrated iot. 
IEEE transactions on industrial informatics 17(4):2871-2879

20. Molano JIR, Lovelle JMC, Montenegro CE, Granados JJR (2018) Crespo RG Ruben Metamodel for integration of Internet of Things. Social Networks the Cloud Industry 4:709-723

21. Amudha G, Narayanasamy P (2018) Distributed location and trust based replica detection in wireless sensor networks. Wireless Pers Commun 102(4):3303-3321

22. Molano JIR, Lovelle JMC, Montenegro CE, Granados JJR, Crespo RG (2018) Metamodel for integration of internet of things, social networks, the cloud and industry 4.0. Journal of ambient intelligence humanized computing 9(3):709-723

23. Younan M, Houssein EH, Elhoseny M, Ali AA (2020) Challenges and recommended technologies for the industrial internet of things: A comprehensive review. Measurement 151:107198

24. Goad D, Collins AT, Gal U (2021) Privacy and the Internet of Things - An experiment in discrete choice. Information Management 58(2):103292

25. Fataliyev T, Mehdiyev S (2020) Industry 4.0: The oil and gas sector security and personal data protection. International Journal of Engineering Manufacturing 10(2):1

26. Fernández-Caramés TM, Fraga-Lamas $P$ (2020) Use case based blended teaching of IloTcybersecurity in the industry 4.0 era. Applied Sciences 10(16):5607

27. Patel T, Patel V (2020) Data privacy in construction industry by privacy-preserving data mining (PPDM) approach. Asian Journal of Civil Engineering 21(3):505-515

28. Sharma S, Saini H (2020) Fog assisted task allocation and secure deduplication using 2FBO2 and MoWo in cluster-based industrial loT (IIoT). Comput Commun 152:187-199

29. Kounoudes AD, Kapitsaki GM (2020) A mapping of IoT user-centric privacy preserving approaches to the GDPR. Internet of Things 11:100179

30. Latif S, Idrees Z, Ahmad J, Zheng L, Zou Z (2021) A blockchain-based architecture for secure and trustworthy operations in the industrial Internet of Things. Journal of Industrial Information Integration 21:100190

\section{Figures}




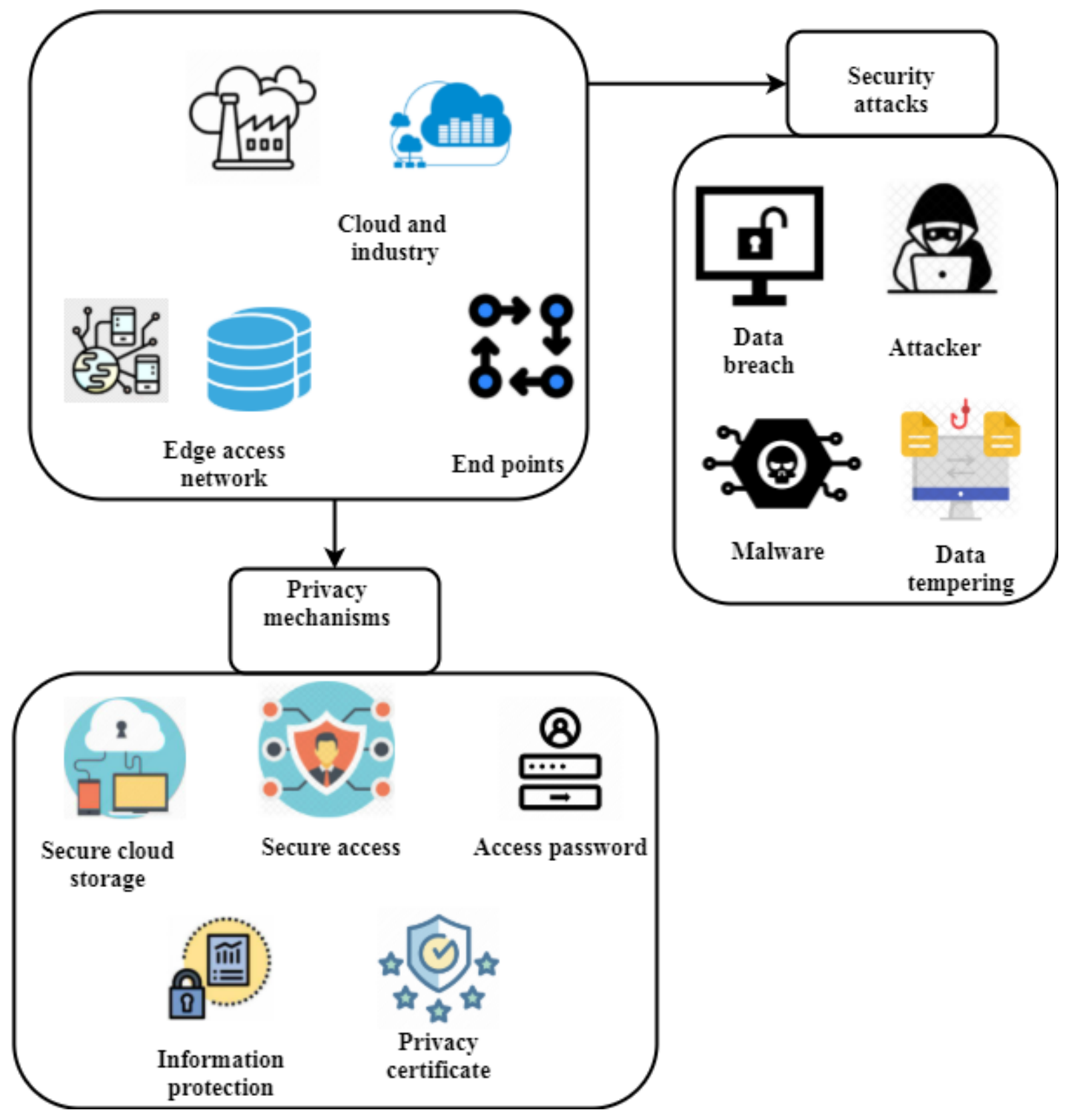

Figure 1

Data security attacks in the loT industry 


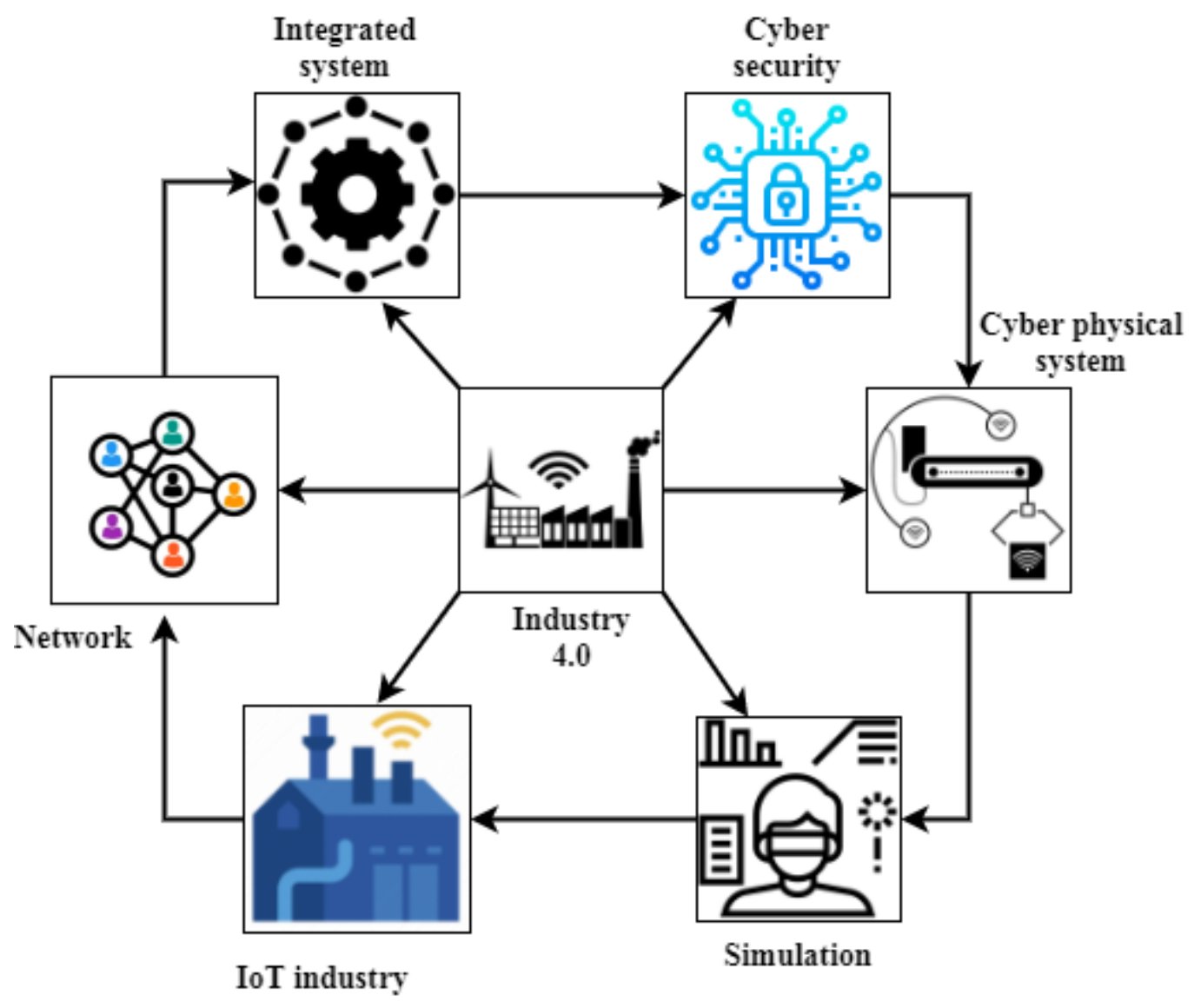

Figure 2

The components of Industry 4.0 


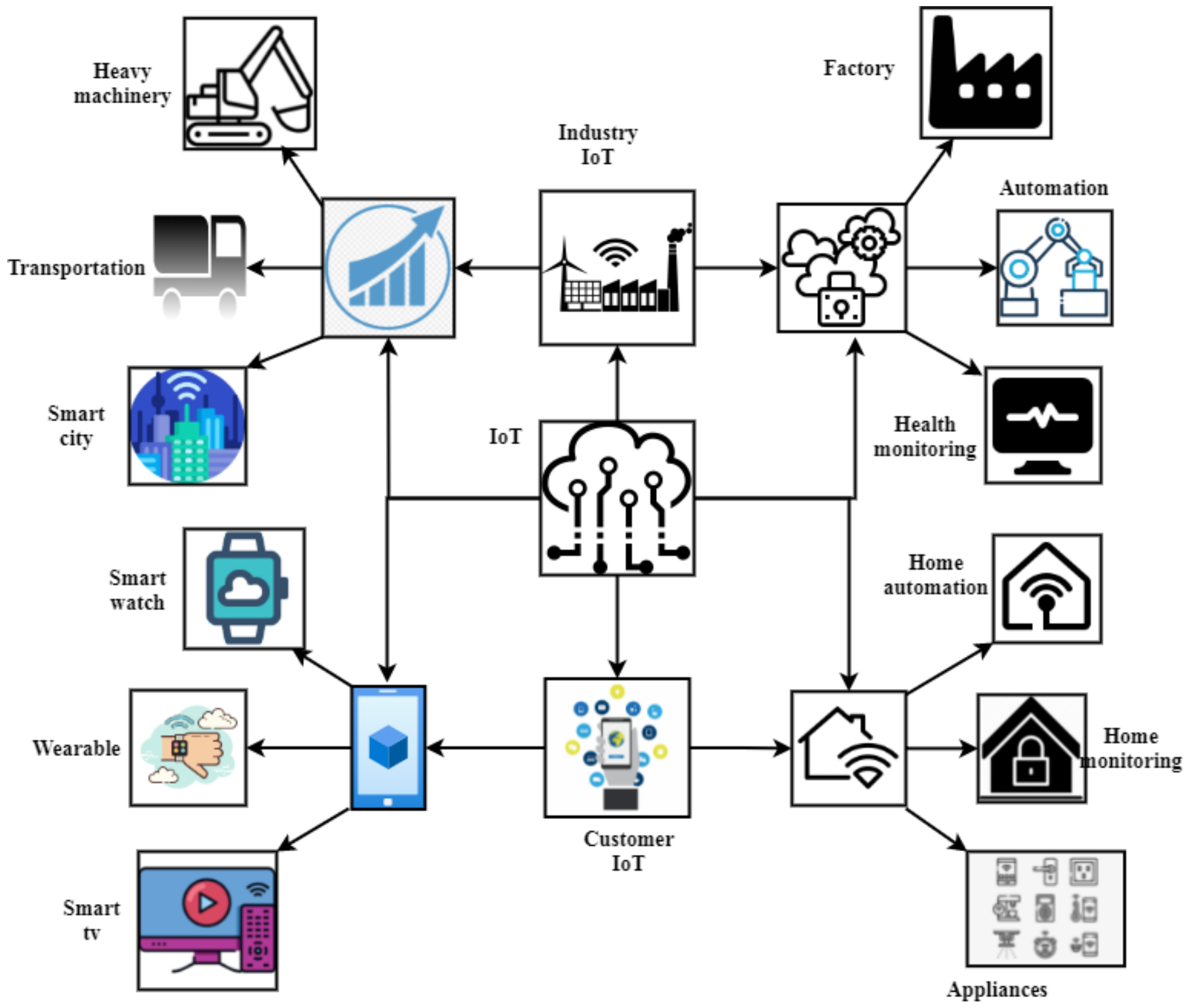

Figure 3

Implications for the Internet of Things 


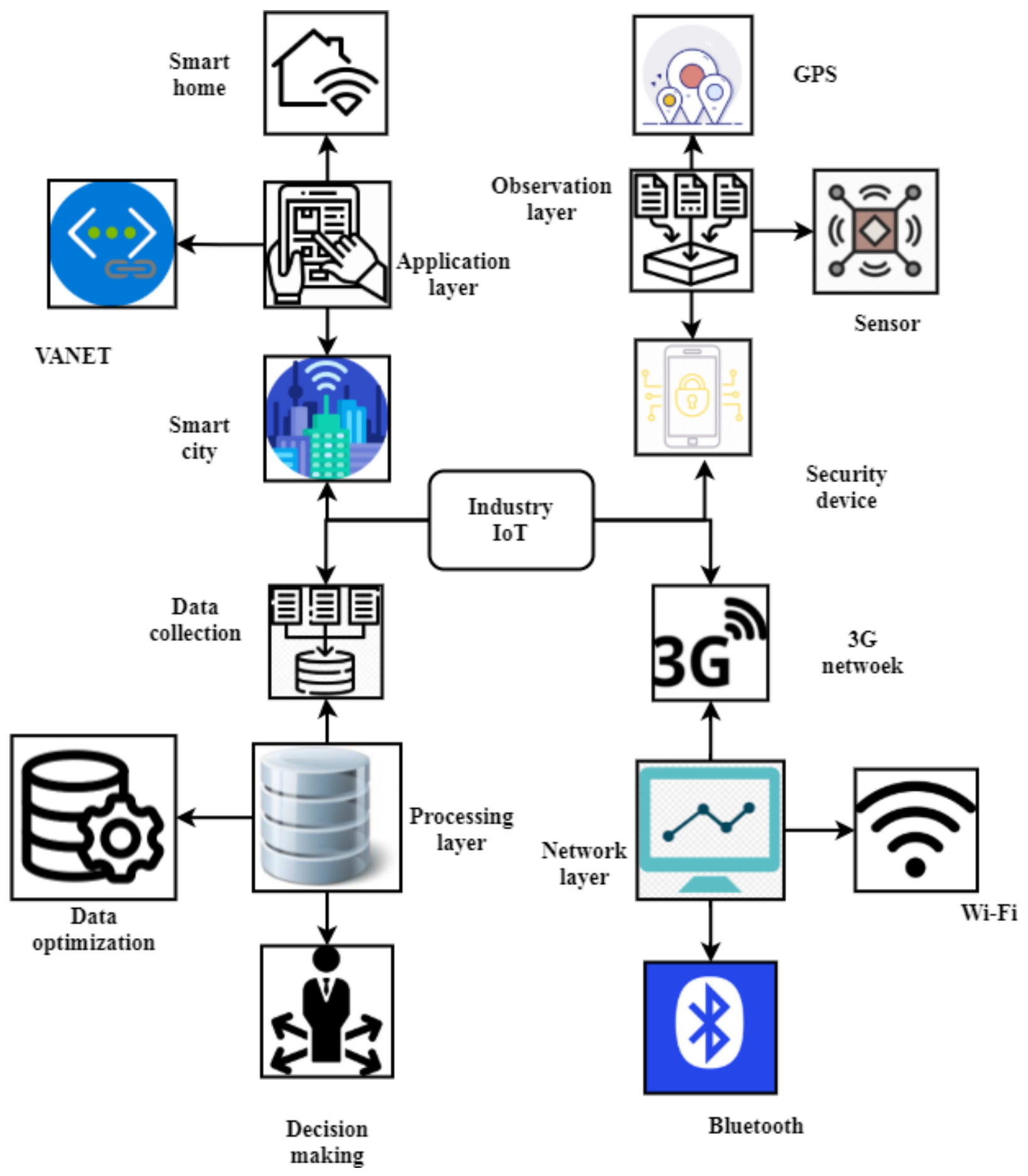

Figure 4

Design of the industrial Internet of Things loT 


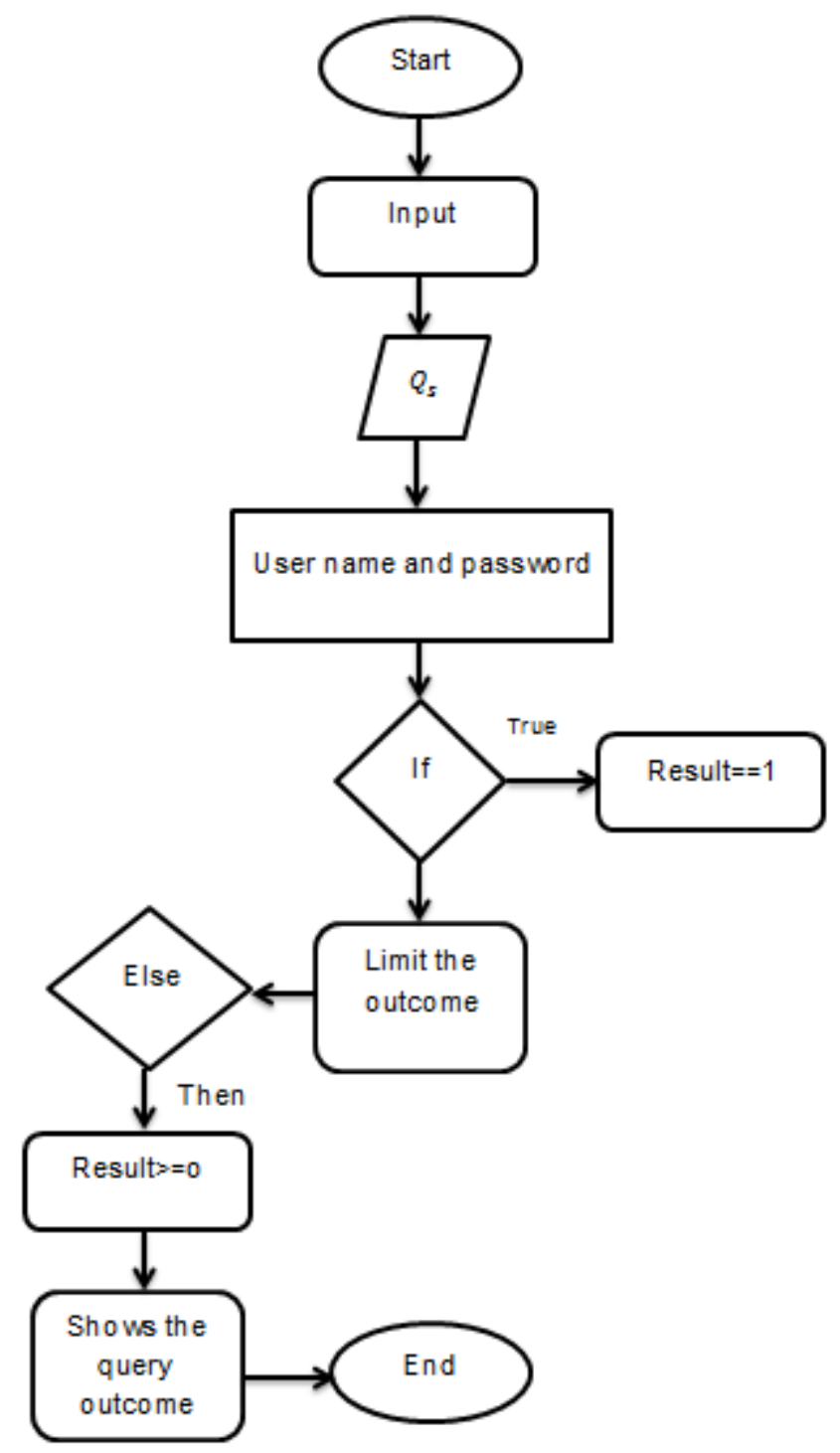

Figure 5

Flow chart for data access control algorithm 


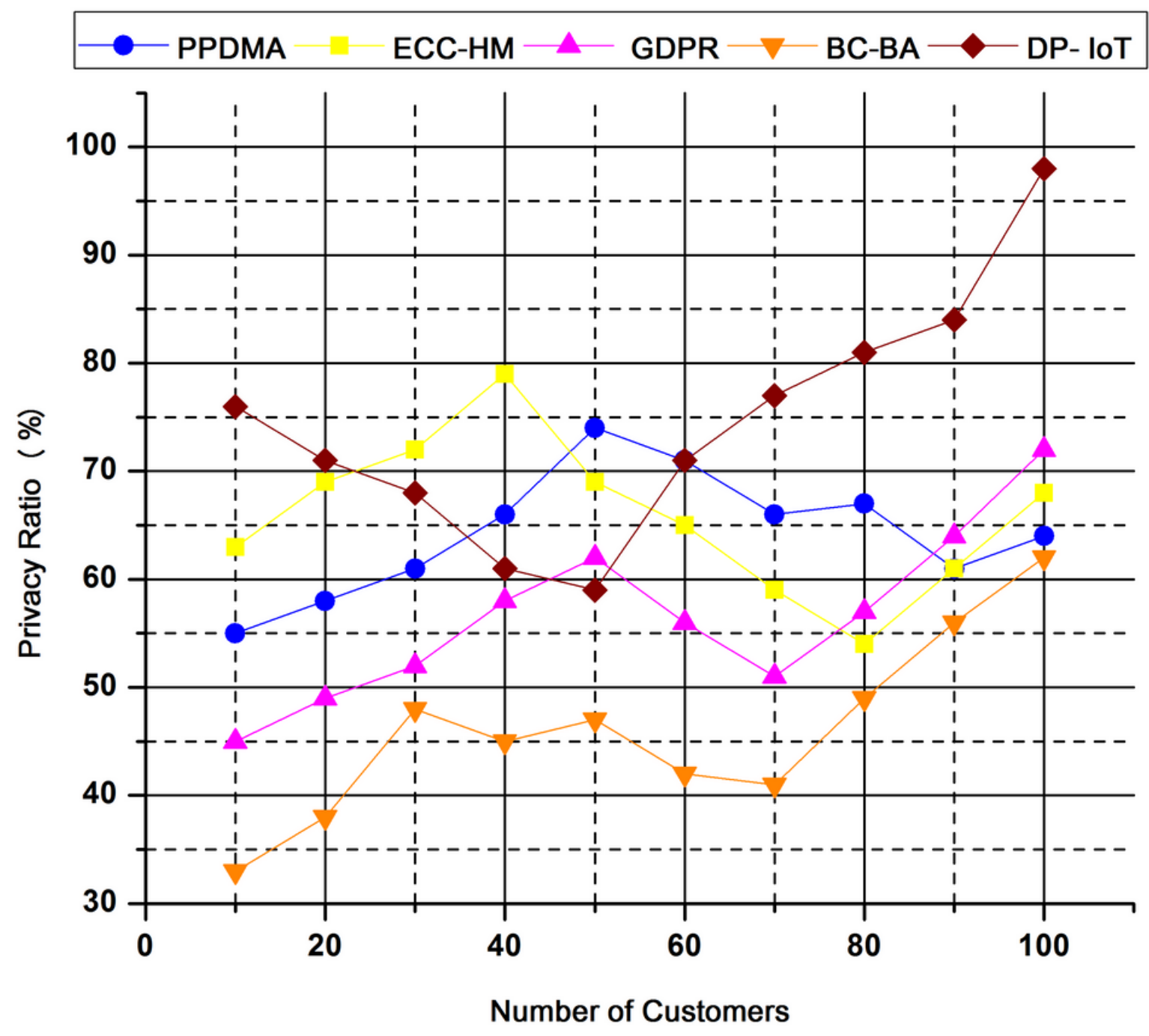

Figure 6

Privacy Ratio (\%) 

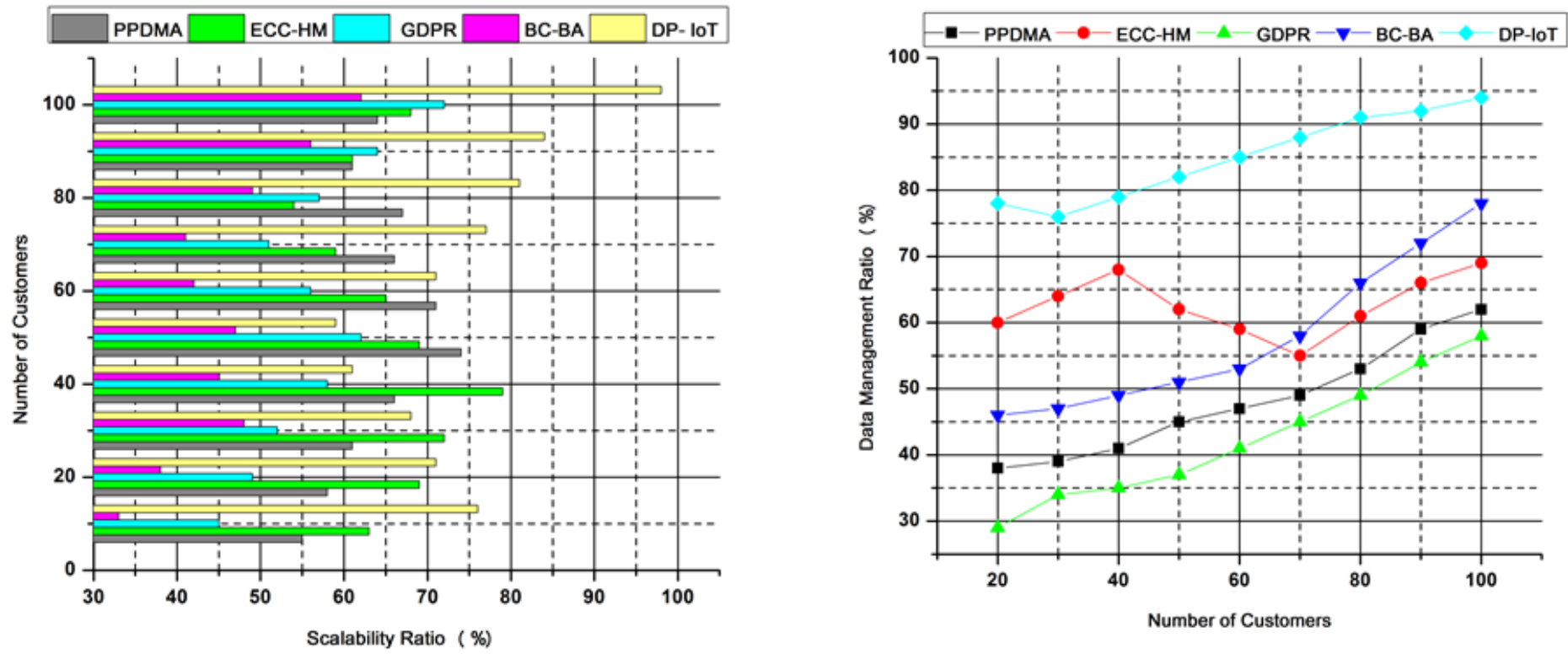

Figure 7

(a)Scalability Ratio (\%) and figure 7 (b) Data Management Ratio (\%)
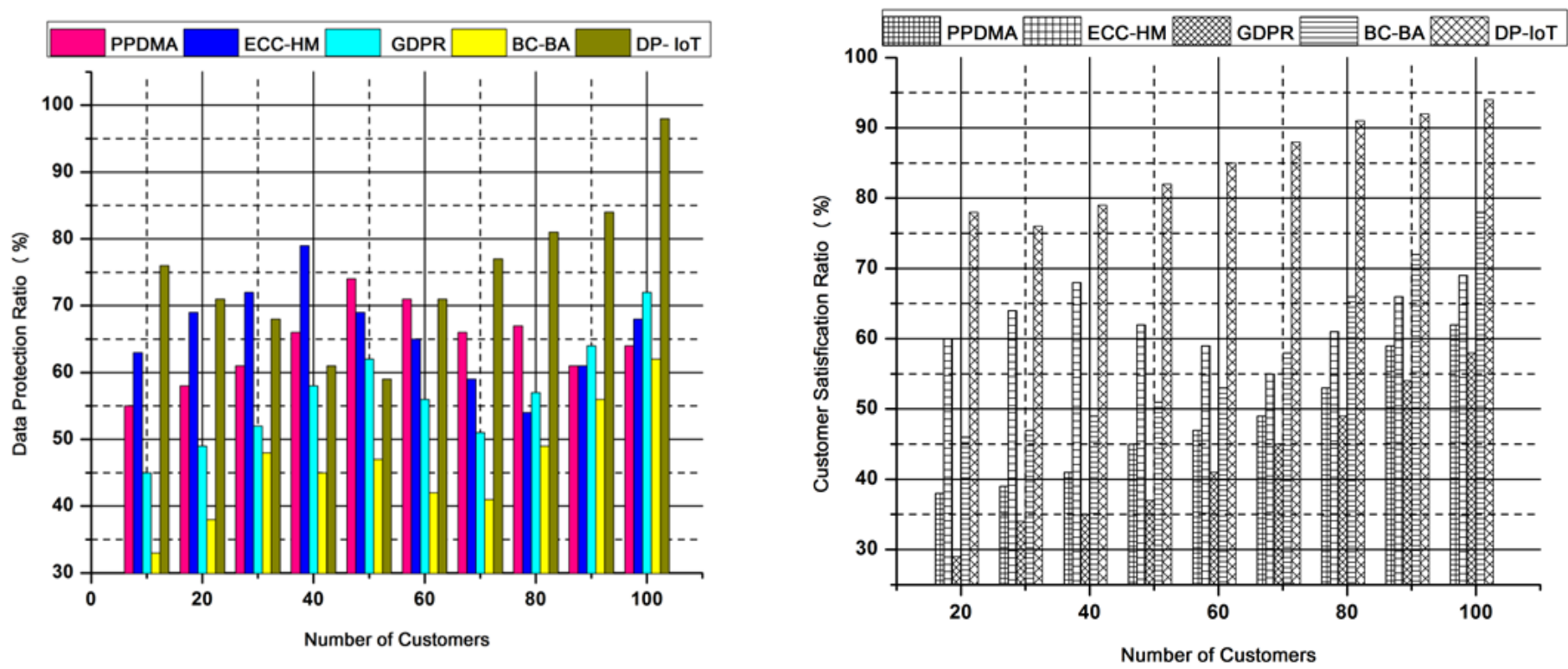

Figure 8

(a) Data Protection Ratio (\%) and figure 8 (b) Customer Satisfaction Rate (\%) 